\title{
Pattern and Appropriateness of Medicines Prescribed to Outpatients at a University Hospital in Northwestern Ethiopia
}

\author{
Fitsum Sebsibe Teni, ${ }^{1}$ Sewunet Admasu Belachew, ${ }^{2}$ \\ Begashaw Melaku Gebresillassie, ${ }^{2}$ Eshetie Melese Birru, ${ }^{3}$ Befikadu Legesse Wubishet, ${ }^{4}$ \\ Bethelhem Hailu Tekleyes, ${ }^{5}$ Bilal Tessema Yimer, ${ }^{5}$ and Yonas Getaye Tefera ${ }^{2}$ \\ ${ }^{1}$ Department of Pharmaceutics and Social Pharmacy, School of Pharmacy, College of Health Sciences, Addis Ababa University, \\ Addis Ababa, Ethiopia \\ ${ }^{2}$ Department of Clinical Pharmacy, School of Pharmacy, College of Medicine and Health Sciences, University of Gondar, \\ Gondar, Ethiopia \\ ${ }^{3}$ Department of Pharmacology, School of Pharmacy, College of Medicine and Health Sciences, University of Gondar, Gondar, Ethiopia \\ ${ }^{4}$ Research Center for Generational Health and Ageing, Faculty of Health and Medicine, University of Newcastle, \\ Callaghan, NSW, Australia \\ ${ }^{5}$ Department of Pharmaceutics, School of Pharmacy, College of Medicine and Health Sciences, University of Gondar, Gondar, Ethiopia
}

Correspondence should be addressed to Fitsum Sebsibe Teni; fitse4@gmail.com

Received 18 July 2017; Accepted 26 November 2017; Published 18 December 2017

Academic Editor: Kurt G. Naber

Copyright (C) 2017 Fitsum Sebsibe Teni et al. This is an open access article distributed under the Creative Commons Attribution License, which permits unrestricted use, distribution, and reproduction in any medium, provided the original work is properly cited.

\begin{abstract}
The study assessed the pattern and appropriateness of medicines prescribed to outpatients at Gondar University Referral Hospital in northwestern Ethiopia. An institution-based cross-sectional study was employed, through interviews and prescription reviews, among 346 patients at the outpatient pharmacy, from 2nd to 20th of May 2016. Data on sociodemographic profile of patients and medicines prescribed to them were collected. A mean of 1.72 medicines per encounter was prescribed, over a third of the total being anti-infectives. Patients were able to get about $85 \%$ of these medicines. An unskilled government employee would be required to work more than one and a half day to be able to afford the average priced medicine. Among prescriptions with two or more medicines, more than a third had at least one potential drug-drug interaction (PDDI), the commonest pair containing amoxicillin and doxycycline. Being male, being older (50-59 years), and increased number of medicines were associated with higher likelihood of PDDIs. In conclusion, the number of medicines prescribed per encounter was up to accepted standard. However, their availability fell short, together with considerable cost. Regarding appropriateness, a significant proportion of potential drug-drug interactions is identified and associated with patient's sex, age, and number of medicines prescribed.
\end{abstract}

\section{Introduction}

Globally, medicine use is expected to reach 4.5 trillion doses in 2020 with the cost predicted being 1.4 trillion dollars. In the same year, over half of the global population will use more than one dose of medicine per person per day. Two-thirds of the medicines in the market will be used in emerging markets, India, China, Brazil, and Indonesia being the leading ones. An improvement in access to medicines globally is also expected, with differences still existing among countries [1].

United Nations Millennium Development Goals related findings reported the availability of medicines in developing countries to be low, with an average of $34.9 \%$ in the public sector of 27 developing countries. Similarly, availability in private sectors was found to be $36.8 \%$ [2]. Another study in Ghana, Kenya, and Uganda shows varying levels of availability among the countries and different levels of health institutions in the countries [3].

As to appropriateness, a World Health Organization (WHO) document on appropriate/rational prescribing outlines a six-step approach to the process. These include defining the problem with the patient, setting objectives regarding its treatment, assessing whether $\mathrm{p}$-drugs/treatments could 
treat it (medicines physicians commonly prescribe and are familiar with), initiating the treatment, providing information, instruction, and warnings, and monitoring/stopping the treatment [4]. Another article which added two steps to the above process included consideration of cost of medicines and employing computer and other tools to minimize the occurrence of prescribing errors [5].

Looking at medicine use in Africa, a systematic review of studies done in eleven countries on medicine prescribing practice spanning over twenty years period reported poor prescribing practice. Compared against WHO's criteria, the review pointed out that high level of antibiotics and injections were prescribed. The review noted that the findings were worse compared to studies from other regions [6].

Studies on potential drug-drug interaction (PDDI) in a number of countries reported varying findings. Studies from India and Sri Lanka, which employed Medscape Interaction Checker, reported PDDIs proportions of $83.42 \%$ and $52.5 \%$ $[7,8]$. Findings from Brazil and Italy, using Drug Reax of Micromedex, identified $49.7 \%$ and $45.3 \%$ pairs of medicines with PDDIs $[9,10]$.

In Ethiopia, a number of studies assessed availability, affordability, and appropriateness. Among these, there were studies on availability and prices of medicines in the public and private sectors [11]. Similarly, another study focused on availability of medicines at health centers in rural Ethiopia, in relation to status of protection by fee waiver system to patients from costs due to medicines [12]. Another assessment of availability and affordability of essential medicines for children in the western part of Ethiopia reported levels of $43 \%$ and $42.8 \%$ in public and private sectors, respectively [13].

As to the pattern of medicine prescribing, some studies reported a range of findings from different parts of the country [14-19]. A few studies on appropriateness of medicine prescription, in the form of assessing PDDIs, were conducted in Ethiopia. These focused on a range of patient categories including pediatrics, elderly, outpatients, and inpatients. Considerable proportions of prescription of PDDIs were reported by many of these studies [20-24].

Despite the above literature, studies assessing the pattern and appropriateness of medicines prescribed to outpatients remain scarce in providing information to stakeholders for possible actions. This study aimed to assess the pattern and appropriateness of medicines prescribed to outpatients visiting Gondar University Referral Hospital (GURH) in northwestern Ethiopia.

\section{Materials and Methods}

2.1. Study Setting and Period. This study was conducted at Gondar University Referral Hospital (GURH) from 2 to 20 May, 2016. It is a referral and teaching hospital with a catchment population of five million. It provides services in several departments including obstetrics and gynecology, internal medicine, surgery, and pediatrics. Inpatient as well as outpatient pharmacy services are also provided in the hospital.
2.2. Study Design. An institution-based cross-sectional study was conducted among adult outpatients visiting the hospital outpatient pharmacy. This was undertaken through interview with patients at the pharmacy when coming to collect prescribed medicines and reviewing their prescriptions.

2.3. Sampling. This manuscript presents the pattern and appropriateness of prescribed medicines; it is part of a project which focused on assessing pattern of medicines prescribed and costs incurred by patients and their families during outpatient visits to the hospital. The sample size determination was made based on estimate of costs incurred by patients. Published mean and standard deviation (SD) of cost for general outpatient visits were not found from previous studies in Ethiopia, to the best of literature search done. So, sample size calculation was done using the single population mean formula through making reasonable estimates of the incurred minimum and maximum costs. These were used to predict standard deviation (SD) which was taken to be a quarter of the range [25-27].

The cost range estimate was from 25 Ethiopian Birr (ETB) (a little more than 1 USD) to a maximum of 500 ETB (about 22 USD), SD being 118.75 ETB. The formula $\left[N=\left(\left(z_{1-\propto}\right)^{2} \times\right.\right.$ $\left.\sigma^{2}\right) / \delta^{2}$, is used to calculate the sample size, where $z_{1-\propto}$ was set as 1.96 at $95 \%$ confidence interval (CI), with a standard deviation $(\sigma)$ of 5.44 USD (118.75 ETB), and margin of error $(\delta)$ set at 5\% (0.6 USD (13.12 ETB)). This provided a sample size of 314.5 and with a $10 \%$ contingency; the total sample size was calculated to be 346 .

In the selection of participants of the study, every fifth patient receiving service at the outpatient pharmacy was approached, in each of the fifteen weekdays during the data collection period by dividing total sample by the number of days. This was done to increase the representativeness of the sample of outpatients visiting the hospital.

2.4. Data Collection. The data collection instrument contained sections on sociodemographic variables and medicines prescribed. It was pretested on prescriptions for 30 patients before the actual data collection which were not included in the final analysis.

Data collection was conducted from 2 to 20 May, 2016, by four pharmacy students. Interviews with patients and review of prescriptions they brought to the outpatient pharmacy were conducted. The data collectors were provided with one-day training on the data collection, instrument, and communication with respondents.

2.5. Data Entry, Analysis, and Interpretation. The data collected were entered into and analyzed with Statistical Packages for Social Sciences version 23. Mean, median, and proportion have been employed for descriptive analysis, presented using tables and a graph. Binary logistic regression test was done to identify predictors of the occurrence of PDDIs; the results are presented using crude and adjusted odds ratio [OR]. In this analysis a 95\% confidence interval (CI) with a $p$ value of 0.05 was used as cut-off point to determine statistical significance of associations. 
The medicines identified from the prescriptions for patients in the study were classified into level one Anatomical Therapeutic Chemical (ATC) groups [28]. Availability of medicines was calculated using the proportion of total number of medicines patients were able to get from the pharmacy to the total number of medicines prescribed. In assessing the affordability of medicines among patients, the average spending per medicine was divided by the daily wage of the lowest paid government worker [29].

The presence of potential drug-drug interactions (PDDIs) was assessed using Medscape Drug Interaction Checker, an online interaction checking platform [30]. The levels, "Serious-Use Alternative," "Monitor Closely," and "Minor," were used to classify the PDDIs found among the medicines prescribed.

2.6. Ethical Considerations. The study was approved by the Ethical Review Committee of School of Pharmacy at the College of Medicine and Health Sciences, University of Gondar. In the process of data collection, the consent of each of the participants of the study was sought. The collected data were kept strictly confidential and used only for the purposes of the study.

\section{Results}

Of the 346 interview encounters and prescription reviews, 342 were included in the final analysis with the remaining removed due to incompleteness. Most of the participants in the study were female patients (61.1\%) and were under the age of $30(41.2 \%)$. More than a third (33.9\%) of the participants reported that they were unable to read and write. In terms of occupation, a comparable proportion (35.1\%) reported to having the role of housewife. The majority of the individuals in the study (69.0\%) earned less than 500 ETB (22.9 USD). Two-thirds (64.9\%) of them were also from outside the town of Gondar (Table 1).

In terms of the nature of patients' visit to the hospital, the majority came for treatment of a chronic illness or a followup visit, which accounted for two-thirds. The remaining came due to acute illness.

3.1. Medicines Prescribed to Patients. The outpatients visiting the hospital were prescribed 106 different medicines, with a total frequency of 588 medicines. This was calculated to be 1.72 medicines per patient on average. Looking at the type of medicines prescribed, anti-infectives for systemic use were the most frequent level one ATC groups of medicines, a third $(33.5 \%)$ of the medicines prescribed. Medicines for nervous system (15.0\%) and those for alimentary tract and metabolism (11.6\%) followed anti-infectives in their frequency (Figure 1).

The ten most frequently prescribed medicines accounted for nearly half (44.7\%) of the total, amoxicillin and omeprazole being the most common ones (Table 2).

3.2. Availability and Affordability of the Prescribed Medicines. Of the total number of medicines prescribed, patients were able to get their prescriptions filled for the 496 medicines
TABLE 1: Sociodemographic profile of participants at GURH, 2016.

\begin{tabular}{|c|c|}
\hline Variable & Frequency (\%) \\
\hline \multicolumn{2}{|l|}{ Age (years) } \\
\hline $18-29$ & $141(41.2)$ \\
\hline $30-39$ & $84(24.6)$ \\
\hline $40-49$ & $46(13.5)$ \\
\hline $50-59$ & $45(13.2)$ \\
\hline $60+$ & $26(7.6)$ \\
\hline \multicolumn{2}{|l|}{ Gender of the patient } \\
\hline Male & $133(38.9)$ \\
\hline Female & $209(61.1)$ \\
\hline \multicolumn{2}{|l|}{ Marital status } \\
\hline Married & $195(57.0)$ \\
\hline Unmarried & $100(29.2)$ \\
\hline Divorced/separated & $27(7.9)$ \\
\hline Widow/er & $20(5.8)$ \\
\hline \multicolumn{2}{|l|}{ Educational status } \\
\hline Unable to read and write & $116(33.9)$ \\
\hline Able to read and write & $33(9.6)$ \\
\hline Primary school (Grades 1-8) & $72(21.1)$ \\
\hline Secondary school (9-10) & $55(16.1)$ \\
\hline College preparatory level & $14(4.1)$ \\
\hline Technical and vocational education and training & $31(9.1)$ \\
\hline University education & $21(6.1)$ \\
\hline \multicolumn{2}{|l|}{ Major occupation } \\
\hline Government employee & $36(10.5)$ \\
\hline Private company employee & $18(5.3)$ \\
\hline Self-employed/business person & $16(4.7)$ \\
\hline Housewife & $120(35.1)$ \\
\hline Farmer & $76(22.2)$ \\
\hline Student & $43(12.6)$ \\
\hline Unemployed & $21(6.1)$ \\
\hline Other $^{a}$ & $12(3.5)$ \\
\hline \multicolumn{2}{|l|}{ Permanent residence } \\
\hline Gondar town & $120(35.1)$ \\
\hline Areas outside Gondar town & $222(64.9)$ \\
\hline \multicolumn{2}{|l|}{ Monthly income } \\
\hline Up to 500 ETB (22.9 USD) & $236(69.0)$ \\
\hline More than 500 ETB (>22.9 USD) & $106(31.0)$ \\
\hline
\end{tabular}

${ }^{a}$ Daily laborer, driver.

(84.4\%). The average amount of money per medicine, patients spent at the dispensary, was 1.45 USD. As to the affordability of the medicines dispensed to them, the average amount of money spent per medicine and the daily wage of lowest paid unskilled government employees (0.87 USD) were used to calculate it. On the basis of this, an unskilled lowest paid government employee needs to work for more than one and a half day (1.67 days) to be able to pay for a medicine prescribed to them.

3.3. Proportion and Predictors of PDDIs among Medicines Prescribed to Patients. Of the 342 patients in the study, 164 were prescribed two or more medicines. Among these prescription 


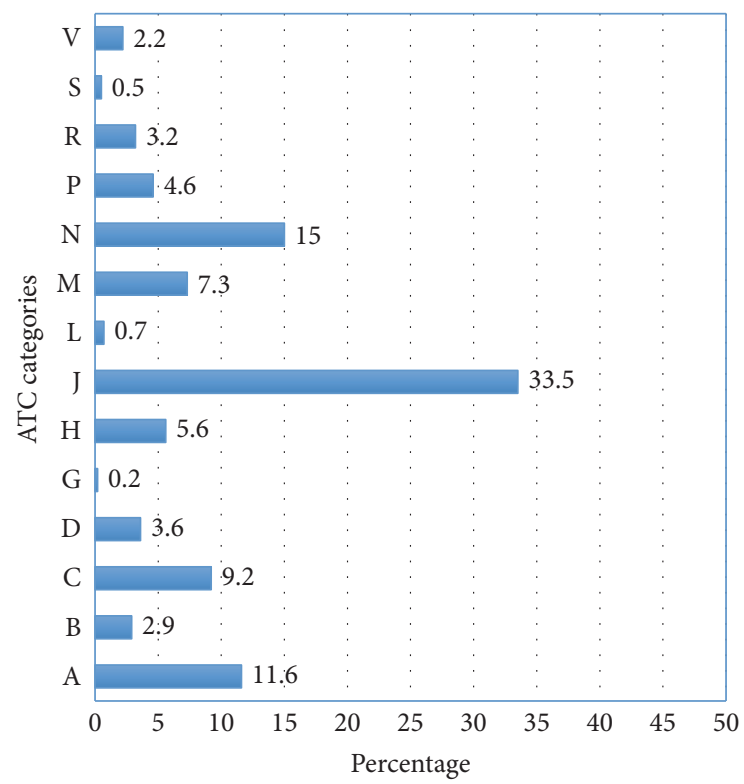

FIGURE 1: ATC level one categories of medicines prescribed to patients. A: alimentary tract and metabolism; B: blood and blood forming organs; C: cardiovascular system; D: dermatologicals; G: genitourinary system and sex hormones; $\mathrm{H}$ : systemic hormonal preparations, excluding sex hormones and insulin; J: anti-infectives for systemic use; L: antineoplastic and immunomodulating agents; $\mathrm{M}$ : musculoskeletal system; N: nervous system; P: antiparasitic products, insecticides, and repellents; R: respiratory system; $\mathrm{S}$ : sensory organs; V: various.

TABle 2: The ten most frequently prescribed medicines at the outpatient department of GURH, 2016.

\begin{tabular}{lcc}
\hline Rank & Name of medicine & Frequency (\%) \\
\hline$(1)$ & Amoxicillin & $47(8.0)$ \\
$(2)$ & Omeprazole & $46(7.8)$ \\
$(3)$ & Metronidazole & $29(4.9)$ \\
$(4)$ & Doxycycline & $24(4.1)$ \\
$(5)$ & Amoxicillin + clavulanic acid & $21(3.6)$ \\
$(6)$ & Ciprofloxacin & $21(3.6)$ \\
$(7)$ & Insulin & $21(3.6)$ \\
$(8)$ & Tramadol & $20(3.4)$ \\
$(9)$ & Diclofenac & $18(3.1)$ \\
$(10)$ & Norfloxacin & $15(2.6)$ \\
\hline
\end{tabular}

encounters, just above a third (34.5\%) were found to have at least one PDDI. Table 3 summarizes the commonest pairs of medicines involved in PDDIs. Amoxicillin and doxycycline coprescription was found to be the most common.

Looking at the severity level of the PDDIs, those requiring close monitoring due to their significance were found to account for more than half (56.31\%) of the total PDDIs identified. PDDIs which were associated with a recommendation at "serious" level contributed the lowest proportion accounting for $13.59 \%$ (Table 4 ).

In terms of the predictors associated with the prescribing of PDDIs, male patients were more than three times likely to be prescribed medicines with PDDIs compared to women $(\mathrm{AOR}=3.168$ [1.320-7.601]). PDDIs were also associated with age, persons in the age group of 50 to 59 years being more than six times more likely $(\mathrm{AOR}=6.457[1.921-21.702])$ to get prescriptions with PDDIs compared to patients in the age groups of 18 to 29 years. The number of medicines prescribed was also found to predict the occurrence of PDDIs, with prescribing of one more medicine being associated with a six times more likelihood of occurrence of PDDIs (AOR $=6.002$ [3.068-11.739]) (Table 5).

\section{Discussion}

The study assessed the pattern of medicines prescribed and their appropriateness in terms of proportion of prescriptions with PDDIs. The average number of medicines prescribed to patients was within the acceptable standard set by WHO [31]. Many of the findings from studies in different parts of Ethiopia showed comparably higher average number of medicines per prescription, ranging from 1.82 to 2.34 [15-19, 32]. Similarly higher number of medicines per prescription were reported by studies from other countries including China, Nigeria, and United Arab Emirates [33-35]. Difference in the composition of cases among patients for which medicines were prescribed could be one of the reasons for the difference.

As to the type of medicines prescribed to outpatients, anti-infectives for systemic use were the most frequent ones. This was in line with the disease burden in the country, where many of the top ranking diseases causing disability or death are of infectious nature [36]. A study from Sri Lanka reported medicines for acid related disorders and antibacterials among the most frequently prescribed. This was similar to the current study, although the top two groups in the cited study were for noninfectious diseases [8]. Other studies in Ethiopia also reported similar findings with antiinfectives prescribed in high proportions [17-20]. Looking at individual medicines, seven of the ten most frequently prescribed medicines were anti-infectives. Among these, amoxicillin was the most frequently prescribed, similar to findings of other studies in different parts of Ethiopia $[15,17$, $18]$.

Of the total number of medicines prescribed to them, patients were able to get about $85 \%$. This was lower compared to the WHO recommended standards [31]. Studies in different parts of Ethiopia reported a range of findings in the different hospitals studied, ranging from as low as about $70 \%$ to $100 \%$ of the prescribed medicines actually dispensed $[15-17,19]$. A study assessing health centers in rural Ethiopia reported a comparable proportion of medicines availability based on the proportion of prescriptions filled [12].

In regard to affordability of medicines, lowest paid government employee needs to work for more than a day and a half to be able to afford a medicine of average price among those prescribed. This was fairly comparable to those from other studies as it took average amount of money spent per medicine into consideration. Other studies from Ethiopia reported a range of 0.3-day to more than one-week duration of wages, for specific medicines [13]. Taking the 
TABLE 3: The five most frequent pair of medicines involved in PDDIs, GURH, 2016.

\begin{tabular}{lccc}
\hline Pair of medicines in PDDIs & Type & Severity & Frequency $(\%)$ \\
\hline Amoxicillin + doxycycline & Pharmacodynamic & Serious-Use Alternative & $8(7.77)$ \\
Aspirin + enalapril & Pharmacodynamic & Serious-Use Alternative & $6(5.82)$ \\
Furosemide + spironolactone & Pharmacokinetic & Monitor Closely & $6(5.82)$ \\
Amoxicillin + clarithromycin & Pharmacodynamic & Minor & $5(4.85)$ \\
Amoxicillin + azithromycin & Pharmacodynamic & Minor & $4(3.88)$ \\
\hline
\end{tabular}

TABLE 4: Level of severity of PDDIs identified among medicines prescribed, GURH, 2016.

\begin{tabular}{lc}
\hline Level of PDDI & Frequency (\%) \\
\hline Serious-Use Alternative & $14(13.59)$ \\
Monitor Closely & $58(56.31)$ \\
Minor & $31(30.10)$ \\
Total & $103(100.0)$ \\
\hline
\end{tabular}

fact that a third of the Ethiopian population lives under poverty line, despite recent improvements in the economy, the spending reported here is significant [37]. Another study from the Philippines reported similarly varying durations, from less than one day to more than four days depending on the price of medicines, for generic ones [38]. A much lower duration ranging from 48 minutes to more than one and a half hour work was found by a study from Fiji [39].

As one of the important indicators of appropriateness, presence of PDDIs among the medicines prescribed to patients was assessed. More than a third of the patients in the study were prescribed at least one pair of medicines with PDDIs. This was a little higher compared to a finding from a study in the US which assessed PDDIs in outpatient settings [40]. However, other studies from Ethiopia and other countries found higher to much higher proportions of PDDIs compared to the current study, reaching up to $78 \%$ $[8,10,21,22,41]$. The difference could be associated with higher number of medicines prescribed per individual in the cited studies and the fact that some were done at inpatient facilities where generally higher number of medicines are prescribed, leading to higher probability of interactions occurring.

Looking at factors related to occurrence of PDDIs, being male was statistically significantly associated with a higher likelihood. In contrast, a study among medicines prescribed for the elderly, conducted in Brazil, identified being female to be associated with occurrence of PDDIs [42]. However, a number of other studies found no statistically significant association in terms of sex of patients [21-24, 41]. Difference in specific conditions/diagnoses between male and female patients could be among the possible reasons. Alternatively, due to the smaller number of prescriptions with two or more medicines and the lower proportion of male patients, random variation might have resulted.

Another variable found to be associated with higher likelihood of prescription of PDDIs was age, specifically those aged from 50 to 59 compared to those between the ages of 18 and 29 years. Higher number of medicines and occurrence of more ailments in older ages could explain the higher likelihood of PDDIs. The finding goes in line with studies from Ethiopia, Brazil, and Serbia, which reported that prescriptions for older patients were associated with higher likelihood of PDDIs [21, 41, 42]. Some other studies reported no statistically significant association.

The mean number of medicines prescribed per prescription was associated with higher likelihood of PDDIs occurring. This was in agreement with the findings from many studies including those from Ethiopia, Brazil, and Serbia $[21-24,41,42]$.

\section{Limitation}

The study considered a duration of one month which may not have covered seasonal variations in prescribing of medicines.

\section{Conclusions}

The study found out that the number of medicines per prescription to be acceptable though their availability fell short of recommended level. In terms of type of medicine, anti-infectives took the highest share among those prescribed. The medicines prescribed were also found to have a considerable cost on patients. As to appropriateness, the study also identified presence of a significant proportion of medicine pairs with PDDIs. Age and number of medicines were found to predict PDDIs.

On the basis of the finding, it is recommended that availing medicines in the dispensary should be given due attention by the hospital to help realize good patient outcome and save patients from costs incurred when buying it outside the hospital. Physicians should consider prescribing alternative medicines in terms of PDDIs, especially when dealing with patients requiring many medicines.

\section{Conflicts of Interest}

The authors declare that there are no conflicts of interest regarding the publication of this paper.

\section{Acknowledgments}

The authors of this paper would like to thank the participants for giving their time to be involve in this study. Mr. Abdulwase 
TABLE 5: Binary logistic test for the predictors of PDDIs, GURH, 2016.

\begin{tabular}{|c|c|c|c|c|}
\hline \multirow{2}{*}{ Variable } & \multicolumn{2}{|c|}{ Presence of PDDI } & \multirow{2}{*}{ Crude OR [95\% CI] } & \multirow{2}{*}{ Adjusted OR [95\% CI] } \\
\hline & Yes $(\%)$ & No $(\%)$ & & \\
\hline \multicolumn{5}{|l|}{ Sex } \\
\hline Male & $28(43.1)$ & $37(56.9)$ & 1.827 [0.949-3.515] & $3.168[1.320-7.601]^{*}$ \\
\hline Female & $29(29.3)$ & $70(70.7)$ & 1 & 1 \\
\hline \multicolumn{5}{|l|}{ Age group (Years) } \\
\hline $18-29$ & $13(22.4)$ & $45(77.6)$ & 1 & 1 \\
\hline $30-39$ & $14(31.8)$ & $30(68.2)$ & $1.615[0.667-3.914]$ & $1.223[0.426-3.516]$ \\
\hline $40-49$ & $8(34.8)$ & $15(65.2)$ & $1.846[0.642-5.312]$ & $2.181[0.562-8.466]$ \\
\hline $50-59$ & $13(56.5)$ & $10(43.5)$ & $4.500[1.607-12.607]^{*}$ & $6.457[1.921-21.702]^{*}$ \\
\hline $60+$ & $9(56.3)$ & $7(43.8)$ & $4.451[1.389-14.264]^{*}$ & $3.972[0.897-17.598]$ \\
\hline \multicolumn{5}{|l|}{ Illness/condition } \\
\hline New/recently occurred & $21(39.6)$ & $32(60.4)$ & 1 & 1 \\
\hline Chronic/follow-up & $36(32.4)$ & $75(67.6)$ & $0.731[0.371-1.442]$ & $0.621[0.263-1.466]$ \\
\hline \multicolumn{5}{|l|}{ Number of medicines } \\
\hline$($ mean $(\mathrm{SD}))$ & 3.09 (1.09) & $2.22(0.48)$ & $4.718[2.645-8.416]^{*}$ & $6.002[3.068-11.739]^{*}$ \\
\hline
\end{tabular}

Mohammed, Mrs. Banchamlak Demamu, Mr. Gashaw Sisay, and Mr. Wudneh Simegn are appreciated for their contribution in the data collection process.

\section{References}

[1] IMS Institute for Healthcare Informatics, Outlook and Implications on Pharmaceuticals and Global Medicine, 2015, http:// www.imshealth.com/en/thought-leadership/ims-institute/reports/global-medicines-use-in-2020.

[2] United Nations, "Access to affordable essential medicines," WHO - Delivering on the Global Partnership for achieving the Millennium Development Goals. MDG Gap Task Force Report, MDG Gap Task Force Report, 2008.

[3] S. H. Masters, R. Burstein, B. Decenso et al., "Pharmaceutical availability across levels of care: Evidence from facility surveys in Ghana, Kenya, and Uganda," PLoS ONE, vol. 9, no. 12, Article ID e0114762, 2014.

[4] World Health Organization, "Guide to Good Prescribing A Practical Manual," http://apps.who.int/medicinedocs/en/d/ Jwhozip23e/.

[5] M. Pollock, O. V. Bazaldua, and A. E. Dobbie, "Appropriate prescribing of medications: an eight-step approach," American Family Physician, vol. 75, no. 2, pp. 231-236, 2007.

[6] R. Ofori-Asenso, P. Brhlikova, and A. M. Pollock, "Prescribing indicators at primary health care centers within the WHO African region: A systematic analysis (1995-2015)," BMC Public Health, vol. 16, no. 1, article no. 724, 2016.

[7] P. S. Patel, D. A. Rana, J. V. Suthar, S. D. Malhotra, and V. J. Patel, "A study of potential adverse drug-drug interactions among prescribed drugs in medicine outpatient department of a tertiary care teaching hospital," Journal of Basic and Clinical Pharmacy, vol. 5, no. 2, pp. 44-48, 2014.

[8] D. Rathish, S. Bahini, T. Sivakumar et al., "Drug utilization, prescription errors and potential drug-drug interactions: An experience in rural Sri Lanka," BMC Pharmacology \& Toxicology, vol. 17, no. 1, article no. 27, 2016.
[9] J. M. Cruciol-Souza and J. C. Thomson, "Prevalence of potential drug-drug interactions and its associated factors in a Brazilian teaching hospital," J. Pharm. Pharm. Sci, vol. 9, no. 3, pp. 427433, 2006.

[10] E. Tragni, M. Casula, V. Pieri et al., "Prevalence of the prescription of potentially interacting drugs," PLOS ONE, vol. 8, no. 10, Article ID e78827, 2013.

[11] M. Ewen, W. Kaplan, T. Gedif et al., "Prices and availability of locally produced and imported medicines in Ethiopia and Tanzania," Journal of Pharmaceutical Policy and Practice, vol. 10, no. 1, article no. 7, 2017.

[12] B. S. Carasso, M. Lagarde, A. Tesfaye, and N. Palmer, "Availability of essential medicines in Ethiopia: An efficiency-equity trade-off?" Tropical Medicine \& International Health, vol. 14, no. 11, pp. 1394-1400, 2009.

[13] E. Sado and A. Sufa, "Availability and affordability of essential medicines for children in the Western part of Ethiopia: Implication for access," BMC Pediatrics, vol. 16, no. 1, article no. 40, 2016.

[14] Z. Desta, T. Abula, A. Gebre-Yohannes, and A. Worku, "Drug prescribing patterns for outpatients in three hospitals in northwest Ethiopia," Ethiopian Journal of Health Development, vol. 16, no. 2, 2002.

[15] A. I. Bilal, E. D. Osman, and A. Mulugeta, "Assessment of medicines use pattern using world health organization's prescribing, patient care and health facility indicators in selected health facilities in eastern Ethiopia," BMC Health Services Research, vol. 16, no. 1, article no. 1414, 2016.

[16] M. Sisay, J. Abdela, Z. Kano, M. Araya, M. Chemdi, and A. Fiseha, "Drug prescribing and dispensing practices in tertiary care hospital of eastern ethiopia: evaluation with world health organization core prescribing and patient care indicators," Clinical \& Experimental Pharmacology, vol. 07, no. 03, 2017.

[17] M. Sisay, G. Mengistu, B. Molla, F. Amare, and T. Gabriel, "Evaluation of rational drug use based on World Health Organization core drug use indicators in selected public hospitals of eastern Ethiopia: A cross sectional study," BMC Health Services Research, vol. 17, no. 1, article no. 161, 2017. 
[18] T. S. Summoro, K. D. Gidebo, Z. Z. Kanche, and E. W. Woticha, "Evaluation of trends of drug-prescribing patterns based on WHO prescribing indicators at outpatient departments of four hospitals in southern Ethiopia," Drug Design, Development and Therapy, vol. 9, pp. 4551-4557, 2015.

[19] K. D. Gidebo, T. S. Summoro, Z. Z. Kanche, and E. W. Woticha, "Assessment of drug use patterns in terms of the WHO patient-care and facility indicators at four hospitals in Southern Ethiopia: a cross-sectional study," BMC Health Services Research, vol. 16, no. 1, pp. 1-8, 2016.

[20] T. Gunasekaran, N. Dejene, V. V. Satyaveni, and M. D. Dhanaraju, "Occurrence of drug-drug interactions in Adama Referral Hospital, Adama city, Ethiopia," Journal of Drug Assessment, vol. 4, no. 1, pp. 19-23, 2015.

[21] B. Srikanth, E. Admassie, W. Hailu, T. Melese, and W. Mequanent, "Extent of poly-pharmacy, occurrence and associated factors of drug-drug interaction and potential adverse drug reactions in Gondar Teaching Referral Hospital, North West Ethiopia," Journal of Advanced Pharmaceutical Technology \& Research, vol. 4, no. 4, p. 183, 2013.

[22] A. S. Bhagavathula, A. Berhanie, H. Tigistu et al., "Prevalence of potential drug-drug interactions among internal medicine ward in University of Gondar Teaching Hospital, Ethiopia," Asian Pacific Journal of Tropical Biomedicine, vol. 4, pp. S204-S208, 2014.

[23] F. Teka, G. Teklay, E. Ayalew, and T. Teshome, "Potential drugdrug interactions among elderly patients admitted to medical ward of Ayder Referral Hospital, Northern Ethiopia: A cross sectional study," BMC Research Notes, vol. 9, no. 1, article no. 431, 2016.

[24] H. Getachew, M. Assen, F. Dula, and A. S. Bhagavathula, "Potential drug-drug interactions in pediatric wards of Gondar University Hospital, Ethiopia: A cross sectional study," Asian Pacific Journal of Tropical Biomedicine, vol. 6, no. 6, pp. 534-538, 2016.

[25] S. K. Lwanga, S. Lemeshow, and W. H. Organization, Sample size determination in health studies?: a practical manual, Dt'ermination de la taille d' un čhantillon dans les t'udes sanomt'riques, manuel pratique, 1991.

[26] WHO, "Sampling methods and sample size," in Health Research Methodology: A Guide for Training in Research Methods, p. 77, WHO Regional Office for the Western Pacific, Manila, 2nd edition, 2001.

[27] Exchange Rates UK, Ethiopian Birr (ETB) to US Dollar (USD) exchange rate history, http://www.exchangerates.org.uk/ ETB-USD-exchange-rate-history-full.html.

[28] WHO Collaborating Centre for Drug Statistics Methodology, "Guidelines for ATC classification and DDD assignment 2017," https://www.whocc.no/atc_ddd_index_and_guidelines/guidelines/.

[29] Wage Indicator Foundation, "Minimum Wages in Ethiopia with effect from 01-01-2011," http://www.wageindicator.org/main/ salary/minimum-wage/ethiopia.

[30] Medscape, "Multi-Drug Interaction Checker," http://reference .medscape.com/drug-interactionchecker.

[31] A. Isah, D. Ross-Degnan, J. Quick, R. Laing, and A. Mabadeje, "The development of standard values for the WHO," http:// archives.who.int/icium/icium/posters/1a2_txt.html.

[32] A. A. Desalegn, "Assessment of drug use pattern using WHO prescribing indicators at Hawassa University teaching and referral hospital, south Ethiopia: A cross-sectional study," BMC Health Services Research, vol. 13, no. 1, article no. 170, 2013.
[33] H. Wang, N. Li, H. Zhu, S. Xu, H. Lu, and Z. Feng, "Prescription Pattern and Its Influencing Factors in Chinese County Hospitals: A Retrospective Cross-Sectional Study," PLoS ONE, vol. 8, no. 5, Article ID e63225, 2013.

[34] I. Tamuno and J. O. Fadare, "Drug prescription pattern in a Nigerian Tertiary hospital," Tropical Journal of Pharmaceutical Research, vol. 11, no. 1, pp. 146-152, 2012.

[35] A. Mahmood, A. A. Elnour, A. A. A. Ali, N. A. G. M. Hassan, A. Shehab, and A. S. Bhagavathula, "Evaluation of rational use of medicines (RUM) in four government hospitals in UAE," Saudi Pharmaceutical Journal, vol. 24, no. 2, pp. 189-196, 2016.

[36] Institute for Health Metrics and Evaluation, "Ethiopia," http:// www.healthdata.org/ethiopia.

[37] The World Bank, "Poverty \& Equity Data: Ethiopia," http:// povertydata.worldbank.org/poverty/country/ETH.

[38] Health Action Information Network, "A Survey on Medicine Prices and Availability in the Philippines," 2009, http://haiweb .org/wp-content/uploads/2015/07/A-Survey-on-Medicine-Prices-and-Availability-in-the-Philippines.pdf.

[39] M. Bailey, "Affordability of Medicines," 2004, http://apps.who .int/medicinedocs/en/d/Js18394en/.

[40] J. E. Lafata, L. Schultz, J. Simpkins et al., "Potential drugg-drug interactions in the outpatient setting," Medical Care, vol. 44, no. 6, pp. 534-541, 2006.

[41] B. Nikolic, S. Jankovic, O. Stojanov, and J. Popovic, "Prevalence and predictors of potential drug-drug interactions," Central European Journal of Medicine, vol. 9, no. 2, pp. 348-356, 2014.

[42] P. R. O. Neto, A. Nobili, S. Marusic et al., "Prevalence and predictors of potential drug-drug interactions in the elderly: a cross-sectional study in the Brazilian primary public health system," Journal of Pharmacy \& Pharmaceutical Sciences, vol. 15, no. 2, pp. 344-354, 2012. 


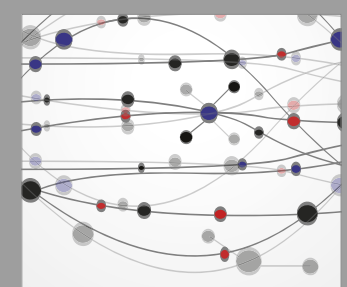

The Scientific World Journal
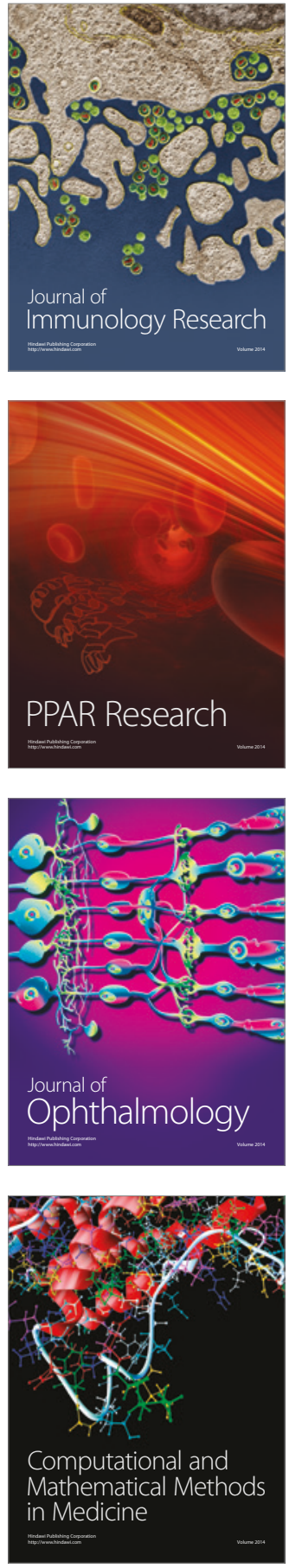

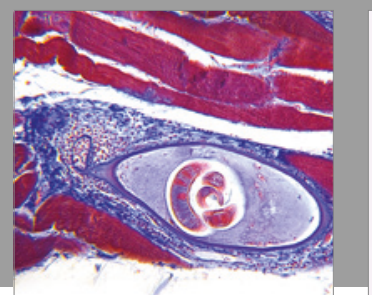

Gastroenterology Research and Practice
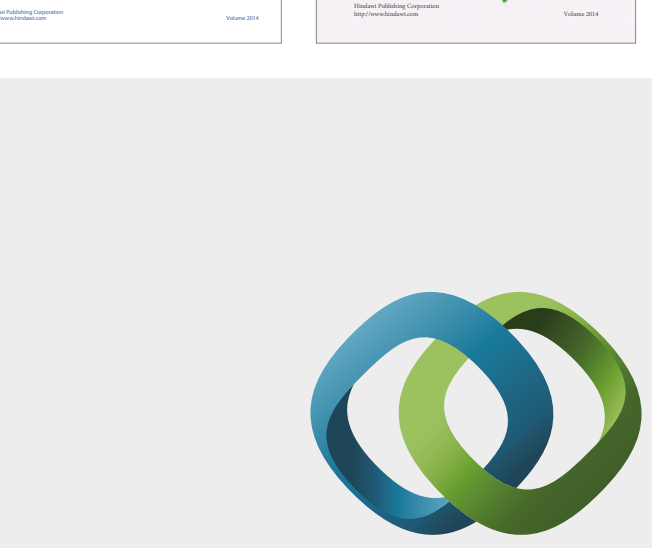

\section{Hindawi}

Submit your manuscripts at

https://www.hindawi.com
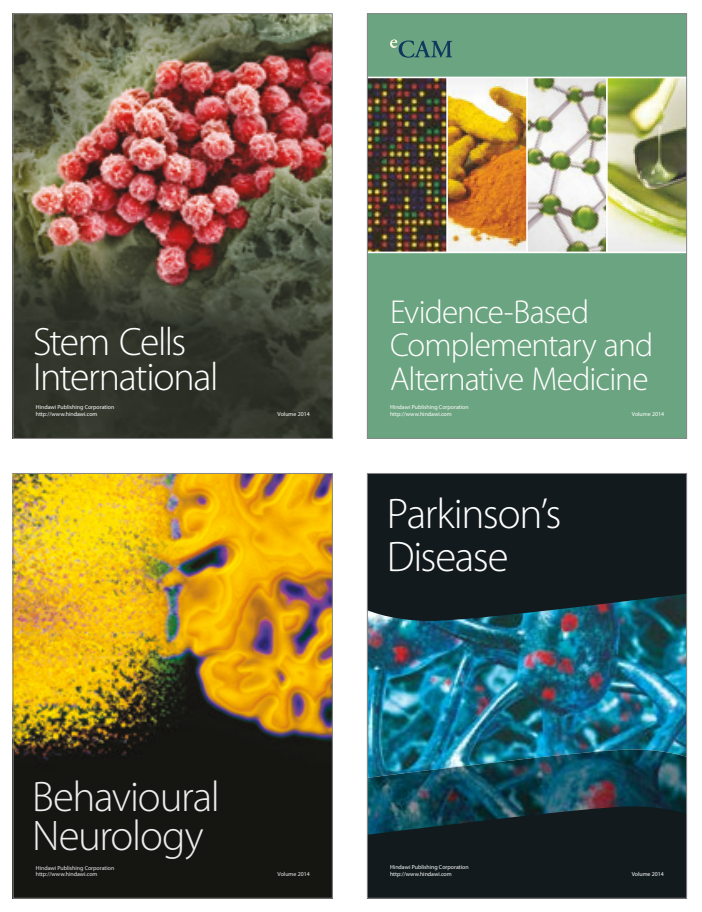
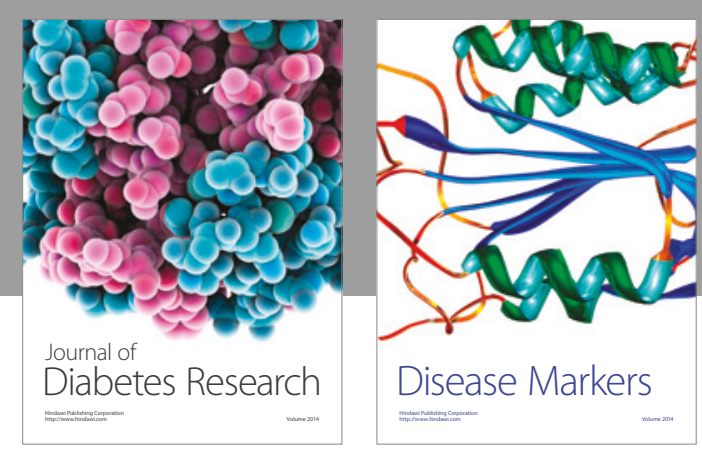

Disease Markers
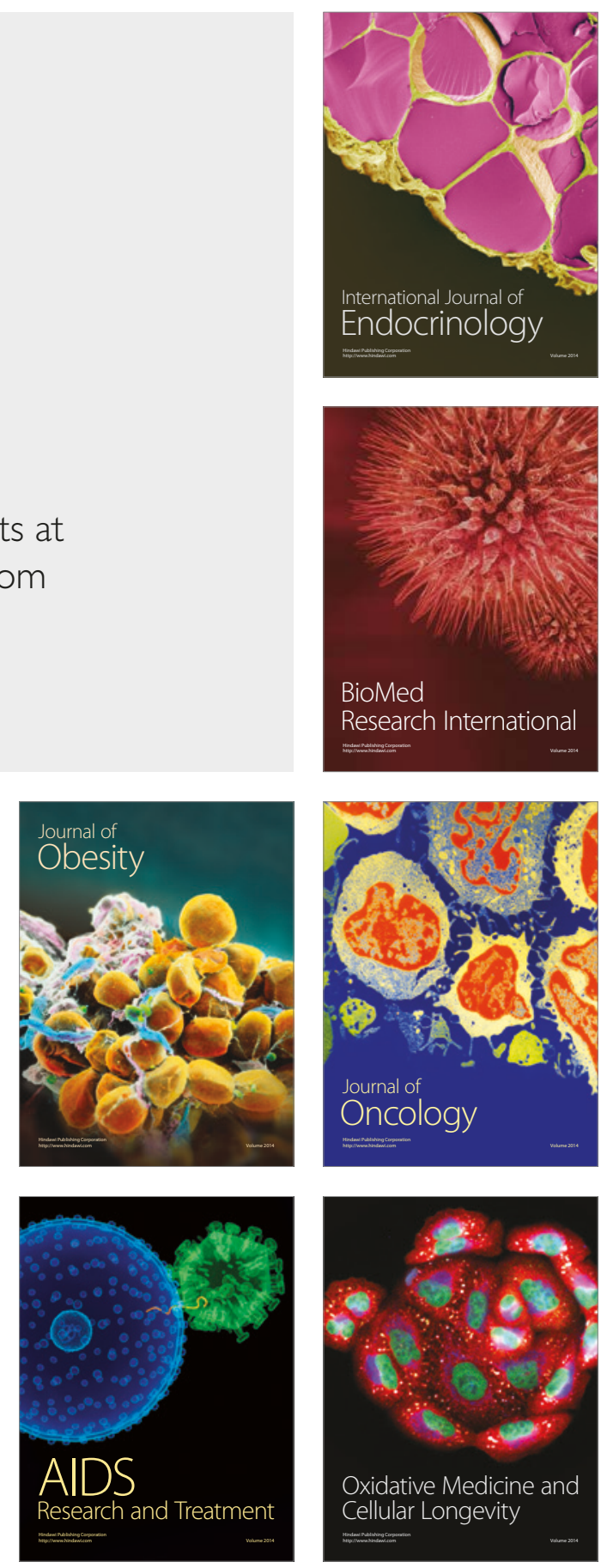